\title{
Turning Model of Tracked Mobile Robots under Steady State Conditions
}

\author{
Jun $\operatorname{Han}^{1, a}$, Guoquan Ren ${ }^{1, b}$, Dongwei $\mathrm{Li}^{1, \mathrm{c}}$ and Ziyong Jia ${ }^{1, \mathrm{~d}}$ \\ ${ }^{1}$ Mechanical Engineering College, Shijiazhuang 050003,China. \\ ahanjun_wy@163.com, ${ }^{b} 709955574 @ q q . c o m$, \\ c12ldw@163.com, ${ }^{\mathrm{d}} 2432282264 @ q q . c o m$
}

Keywords: Tracked Mobile Robots, turning model, steady state, track slipping.

\begin{abstract}
In order to improve the accuracy of motion control, a turning model under steady state conditions of Tracked Mobile Robots (TMRs) is provided, based on considering tracks slipping. With making the sprocket rotating speed ratio of inner track to outer track independent variable, the turning of TMRs under steady state is analyzed and modeled, which is of enormous significance to the motion control of TMRs. And the comparison of numerical analysis results and experimental data shows reliable precision of the model.
\end{abstract}

\section{Introduction}

In recent decades, more and more Tracked Mobile Robots (TMRs) are applied in rescue missions, military reconnaissance, planetary explorations and so on[1,2]. And it is difficult but of great importance to control the motion of TMRs, in the autonomous control and navigation tasks of TMRs. The track slipping effect is inevitable in many practical applications and should be considered necessarily to achieve control purposes[2]. However, researches of TMRs' motion control are mostly based on kinematics model with no slipping[3-5]. In order to improve the accuracy of motion control, a turning model based on conditions of track slipping of TMRs is necessary. Junwei Cheng analyzed the relationship between relative offsets and relative steering radius, based on conditions of track slipping[6]. J Y Wong and C F Chiang gave a general theory for skid steering of tracked vehicles on firm ground[7]. The work done by the authors mentioned above are fundamental, unfortunately, they take steering radius or relative steering radius as the independent variable, which TMRs or other tracked vehicles could not control directly.

In this paper, we address the turning model under steady state of TMRs, based on conditions of track slipping. And in the context of this paper, the sprocket rotating speed ratio of inner track to outer track is applied to independent variable. The ratio is controllable so that the model of turning could be available for TMRs' control. In order to establish the kinematics and dynamics model of TMRs, ignoring secondary factors, it is necessary to make the following assumptions:

(1) TMR is symmetrical about its transverse and longitudinal symmetry plane, and the center of mass coincides with its geometric center, collectively known as the center of the TMR;

(2) The TMR is steering steady on firm road at low speed, excluding the effect of centrifugal force;

(3) Driving resistance coefficient is constant;

(4) The tracks are flexible belt, ignoring the effect of track width;

(5) The track-earth pressure is evenly distributed along the center line of tracks.

\section{Kinematic Analysis}

\subsection{Establish Coordinate System.}

To describe the turning steering of TMRs, corresponding coordinate systems are established. As shown in Fig.1, the xoy coordinate system is follow-up coordinate system fixed on TMRs' center, while $X O Y$ coordinate system is ground coordinate system fixed on the ground. Assume the coordinate systems is coincident in the initial time. The TMR is moving on the $X O Y$ plane, so the position of TMR can be described by $\left(X_{o}, Y_{o}\right)$, the coordinate of TMRs' center and its orientation 
can be described by the heading angle $\theta$, which is the angle between $x$ axis and $X$ axis. To explain easily, the inner track is marked as track 1, while the outer track is marked as track 2.

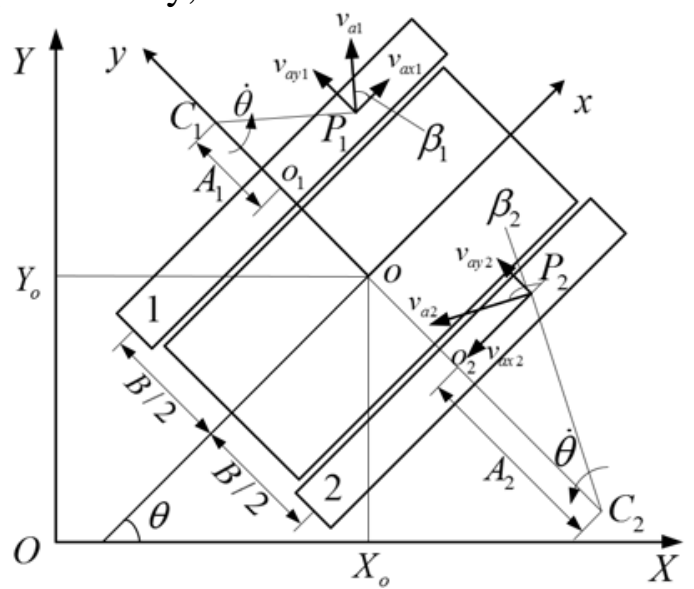

Fig.1 TMR coordinate

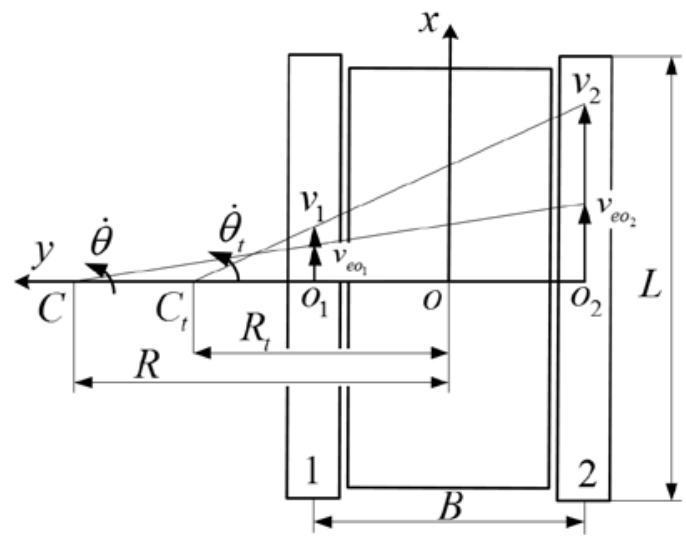

Fig.2 Kinematic analysis of TMR

systems \&. Kinematic analysis of tracks

\subsection{Kinematic Analysis of Tracks.}

In the progress of a TMR turning steering, the instantaneous steering center of the inner and outer track would generate lateral offsets. As shown in Fig.1, point $C_{1}$ is the instantaneous steering center of the inner track, while point $C_{2}$ is the instantaneous steering center of the outer track. And $A_{1}$ is the lateral offset of the instantaneous steering center of the inner track, as well as $A_{2}$ is the lateral offset of the instantaneous steering center of the inner track. $\theta$ is the heading angle, then we can know that $\dot{\theta}$ is the turning angular velocity of TMR and its tracks from theories of plane motion of rigid body. $P_{1}\left(x_{1}, B / 2\right)$ is a point of inner track on track-earth contact area, and $P_{2}\left(x_{2},-B / 2\right)$ is a point of outer track on track-earth contact area, where $B$ is the distance of the center line of tracks. Then we have $x_{1}, x_{2} \in[-L / 2, L / 2]$, where $L$ is the length of track-earth contact area, and the absolute velocities of $P_{1}$ and $P_{2}$ satisfy the equation:

$$
\left\{\begin{array}{l}
v_{a \times 1}=\dot{\theta} A_{1} \\
v_{a \times 2}=\dot{\theta} A_{2}
\end{array}\right.
$$

And the points' velocity components on $y$ axis are:

$$
v_{\text {ayi }}=\dot{\theta} x_{i}
$$

Where $i$ is the marks of tracks. And define the angle of $v_{a i}$, the absolute velocities of $P_{i}$, and positive direction of $x$ axis as $\beta_{i}$, named side-slip angle, then we have:

$$
\left\{\begin{array}{c}
\tan \beta_{1}=\frac{x_{1}}{A_{1}} \\
\tan \beta_{2}=-\frac{x_{2}}{A_{2}}
\end{array}\right.
$$

\subsection{Kinematic Analysis of TMR.}

As shown in Fig.2, when we consider the tracks' slipping, the TMR turns steadily with $C$ as the steering center, $R$ as the turning radius, and $\dot{\theta}$ as the turning angular velocity. Correspondingly, when we do not consider the tracks' slipping, the TMR turns steadily with $C_{t}$ as the steering center, $R_{t}$ as the steering radius, and $\dot{\theta}_{t}$ as the steering angular velocity. Assume the rotating linear velocity of tracks are $v_{1}$ and $v_{2}$, then analyse the velocities of the center points of track-earth contact areas, $O_{1}$ and $O_{2}$, we can get the relationship of velocities with not considering slip: 


$$
\left\{\begin{array}{c}
v_{a o_{1}}=v_{e o_{1}}+v_{r o_{1}}=\dot{\theta}_{t}\left(\mathrm{R}_{t}-\mathrm{B} / 2\right)-v_{1}=0 \\
v_{a o_{2}}=v_{e o_{2}}+v_{r o_{2}}=\dot{\theta}_{t}\left(\mathrm{R}_{t}+\mathrm{B} / 2\right)-v_{2}=0
\end{array}\right.
$$

and the one considering slip:

$$
\left\{\begin{array}{c}
v_{a o_{1}}=v_{e O_{1}}+v_{r O_{1}}=\dot{\theta}(\mathrm{R}-\mathrm{B} / 2)-v_{1}=\dot{\theta} A_{1} \\
v_{a o_{2}}=v_{e o_{2}}+v_{r o_{2}}=\dot{\theta}(\mathrm{R}+\mathrm{B} / 2)-v_{2}=-\dot{\theta} A_{2}
\end{array}\right.
$$

The sprocket rotating speed ratio of inner track to outer track is applied to independent variable. Assume the sprocket rotating speed are $\omega_{1}$ and $\omega_{2}$, the radius of inside and outside caterpillar driving wheel speed, respectively, and the driving gear pitch circle radius of the sprockets are $r$, ignore the driving wheel tooth contact with the track clearance and the tracks' deformation, we can get:

$$
\left\{\begin{array}{l}
v_{1}=\omega_{1} r \\
v_{2}=\omega_{2} r
\end{array}\right.
$$

Define: $\left\{\begin{array}{c}\omega_{2}=\omega \\ k=\omega_{1} / \omega_{2} \\ \lambda=L / B \\ a_{i}=A_{i} /(L / 2)\end{array}\right.$, plug it into equation (4) and (5), then we have no slip model:

$$
\left\{\begin{array}{c}
R_{t}=\frac{1+k}{1-k} \frac{B}{2} \\
\dot{\theta}_{t}=(1-k) \frac{\omega r}{B}
\end{array}\right.
$$

and the slip model:

$$
\left\{\begin{array}{l}
R=\frac{\left(1+\lambda a_{1}\right)+k\left(1+\lambda a_{2}\right)}{1-k} \frac{B}{2} \\
\dot{\theta}=\frac{1-k}{1+\lambda a_{1} / 2+\lambda a_{2} / 2} \frac{\omega r}{B}
\end{array}\right.
$$

Equation (7) and (8) describe two kinematic models, in which the turning radius and angular velocity are functions of speed ratio $k$ and the outer sprocket rotating speed $\omega$. But in the slip model, $R$ and $\dot{\theta}$ are also functions of $a_{1}$ and $a_{2}$. However, $a_{1}$ and $a_{2}$ can not be got from kinematic analysis, here only depend on dynamic analysis.

\section{Dynamic Analysis}

\subsection{Longitudinal Forces of the Tracks.}

When the TMR turns at state of large-radius turning, the braking forces act on the inner track, and the driving forces act on the outer track. The braking forces and driving forces are called longitudinal forces collectively. As shown in Fig.3, By the assumptions, the track-earth pressure is evenly distributed along the center line of tracks, so we can define the unit track-earth pressure along the center line of tracks $q$ as follows:

$$
q=\frac{G}{2 L}
$$




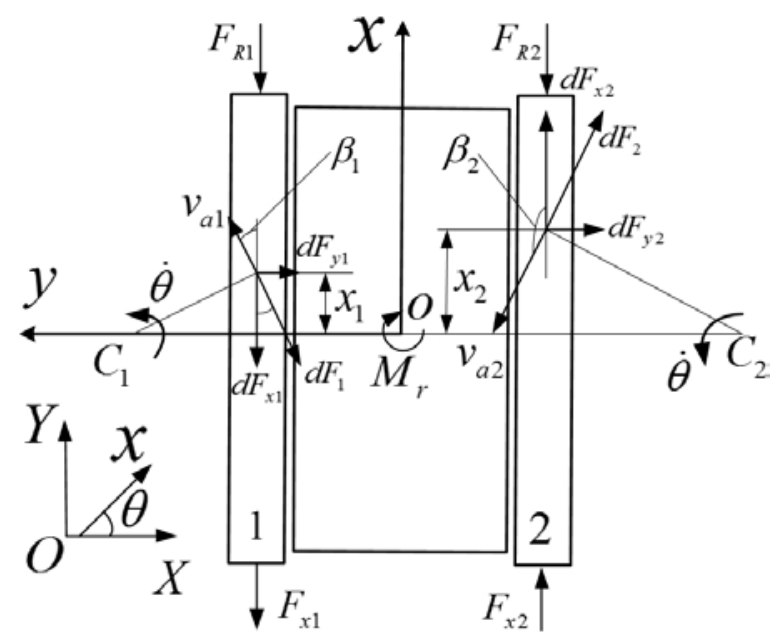

Fig.3 TMR dynamic analysis

Firstly, Consider the longitudinal forces of inner track. When the TMR turns, the inner track rotates the instantaneous steering center $C_{1}$. Take infinitesimal segment $d x_{1}$ at point $P_{1}\left(x_{1}, B / 2\right)$, and the effect force on the infinitesimal segment $d F_{1}$ is proportional to the pressure on infinitesimal segment. The proportionality coefficient is steering resistance coefficient $\mu$. As shown in Fig.3, the longitudinal component of $d F_{1}$ is $d F_{x 1}$, which can be calculated by

$$
d F_{x 1}=-d F_{1} \cos \beta_{1}=-\mu q \cos \beta_{1} d x_{1}
$$

From equation (3) and (9), integrate (10) above the entire track length, we can get the braking force of the inner track:

$$
F_{x 1}=-\int_{-L / 2}^{L / 2} \mu q \cos \beta_{1} d x_{1}=-\int_{-L / 2}^{L / 2} \frac{\mu a_{1} G}{4} \frac{d x_{1}}{\sqrt{\left(a_{1} L / 2\right)^{2}+x_{1}^{2}}}=-\frac{\mu a_{1} G}{4} \ln \frac{\sqrt{a_{1}^{2}+1}+1}{\sqrt{a_{1}^{2}+1}-1}
$$

The minus sign in equation (11) explains the force is opposite to $x$ axis, and the coefficient $\mu$ satisfies equation:

$$
\mu=\mu_{\max } /(0.925+0.15 R / B)
$$

And $\mu_{\max }$ is the steering resistance coefficient of $\mathrm{B} / 2$ turning radius.

Similarly, we can get the driving force of the outer track:

\subsection{Turning Steering Resistance Torque.}

$$
F_{x 2}=\frac{\mu a_{2} G}{4} \ln \frac{\sqrt{a_{2}^{2}+1}+1}{\sqrt{a_{2}^{2}+1}-1}
$$

As shown in Fig.3, the lateral component of $d F_{1}$ is $d F_{y 1}$, which can be calculated by

$$
d F_{y 1}=-d F_{1} \sin \beta_{1}=-\mu q \sin \beta_{1} d x_{1}
$$

And calculate the torque that $d F_{y 1}$ about the rotating center $C_{1}$, we have

$$
d M_{r 1}=-\mu q x_{1} \sin \beta_{1} d x_{1}
$$

From equation (3) and (9), integrate (15) above the entire track length, we can get the steering resistance torque of the inner track:

$$
M_{r 1}=-\int_{-L / 2}^{L / 2} \mu q x_{1} \sin \beta_{1} d x_{1}=-\int_{-L / 2}^{L / 2} \frac{\mu G}{2 L} \frac{x_{1}^{2} d x_{1}}{\sqrt{\left(a_{1} L / 2\right)^{2}+x_{1}^{2}}}=-\frac{\mu G L}{8}\left(\sqrt{a_{1}^{2}+1}-\frac{a_{1}^{2}}{2} \ln \frac{\sqrt{a_{1}^{2}+1}+1}{\sqrt{a_{1}^{2}+1}-1}\right)
$$

The minus sign in equation (16) explains the torque is opposite to the $z$ axis, and the torque makes the TMR rotate clockwise.

Similarly, the steering resistance torque of the outer track is:

$$
M_{r 2}=-\frac{\mu G L}{8}\left(\sqrt{a_{2}^{2}+1}-\frac{a_{2}^{2}}{2} \ln \frac{\sqrt{a_{2}^{2}+1}+1}{\sqrt{a_{2}^{2}+1}-1}\right)
$$


And the total steering resistance torque is:

$$
M_{r}=M_{r 1}+M_{r 2}=-\frac{\mu G L}{8}\left(\sqrt{a_{1}^{2}+1}+\sqrt{a_{2}^{2}+1}-\frac{a_{1}^{2}}{2} \ln \frac{\sqrt{a_{1}^{2}+1}+1}{\sqrt{a_{1}^{2}+1}-1}-\frac{a_{2}^{2}}{2} \ln \frac{\sqrt{a_{2}^{2}+1}+1}{\sqrt{a_{2}^{2}+1}-1}\right)
$$

\subsection{Turning Steering Dynamic Balance Equation of the TMR.}

TMR low-speed uniform turning steering in horizontal plane, ignoring the influence of centrifugal force, the dynamic balance equation of the TMR can be written as:

$$
\left\{\begin{array}{l}
F_{x 1}+F_{x 2}+F_{R 1}+F_{R 2}=0 \\
\left(F_{x 2}-F_{x 1}\right) B / 2+M_{r}=0
\end{array}\right.
$$

In the equation (19) , $F_{R 1}=F_{R 2}=-f G / 2$, and they are moving resistances, while coefficient is $f$. Take the equations (11)(13)(18) into the equation (19), then we can finally get:

$$
\left\{\begin{array}{c}
-\frac{a_{1}}{4} \ln \frac{\sqrt{a_{1}^{2}+1}+1}{\sqrt{a_{1}^{2}+1}-1}+\frac{a_{2}}{4} \ln \frac{\sqrt{a_{2}^{2}+1}+1}{\sqrt{a_{2}^{2}+1}-1}-\frac{f}{\mu}=0 \\
\left(a_{1}+\lambda \frac{a_{1}^{2}}{2}\right) \ln \frac{\sqrt{a_{1}^{2}+1}+1}{\sqrt{a_{1}^{2}+1}-1}+\left(a_{2}+\lambda \frac{a_{2}^{2}}{2}\right) \ln \frac{\sqrt{a_{2}^{2}+1}+1}{\sqrt{a_{2}^{2}+1}-1}-\lambda\left(\sqrt{a_{1}^{2}+1}+\sqrt{a_{2}^{2}+1}\right)=0
\end{array}\right.
$$

Equation (20) gives the relationship of $a_{1}$ and $a_{2}$ between the vehicle parameter $\lambda$ and the ground parameter $f$, as well as a parameter about steering $\mu$.

\section{Numerical Analysis and Experimental Verification}

\subsection{Numerical Analysis.}

Combine equations (8)(12)(20), when the outer sprocket rotating speed $\omega$ is a constant value, the final equations can be rewritten as form:

$$
\left\{\begin{array}{c}
F_{1}\left(a_{1}, a_{2}, R\right)=0 \\
F_{2}\left(a_{1}, a_{2}\right)=0 \\
F_{3}\left(a_{1}, a_{2}, k, R\right)=0
\end{array}\right.
$$

Equations (22) include the lateral relative offsets of instantaneous steering center of tracks $a_{1}$ and $a_{2}$, as well as the turning radius $R$ and the sprocket rotating speed ratio of inner track to outer track $k$, which give the constraint of control variable and motion output variables. $k$ is the control variable, and $a_{1}, a_{2}$, as well as $R$ are motion output variables. Furthermore, equations (22) have no analytical solution, but the numerical solution is solvable. Here we have the related parameters of experimental TMR on the cement floor in Table 1. In addition, the parameters $f$ and $\mu_{\max }$ are measured by experiments.

Table 1 Related Parameters of TMR

\begin{tabular}{cccccc}
\hline$B(m)$ & $L(m)$ & $r(m)$ & $G(N)$ & $f$ & $\mu_{\max }$ \\
\hline 0.505 & 0.520 & 0.072 & 1127 & 0.1508 & 0.65 \\
\hline
\end{tabular}

In the process, we take $\omega=1.7453 \mathrm{rad} / \mathrm{s}$, then we can get the numerical solution of equations (22), by iteration method. When the sprocket rotating speed ratio $k$ is varying from 0 to 1 , the lateral relative offsets of instantaneous steering center of tracks $a_{1}$ and $a_{2}$ is varying as shown in Fig.4 . And when the turning radius $R$ is growing from $B / 2=0.2525 \mathrm{~m}$, the lateral offsets of instantaneous steering center of tracks $A_{1}$ and $A_{2}$ is varying as shown in Fig.5 . These two figures show different sides of the variation of the tracks' lateral offsets, when TMR is turning at different steady states. 


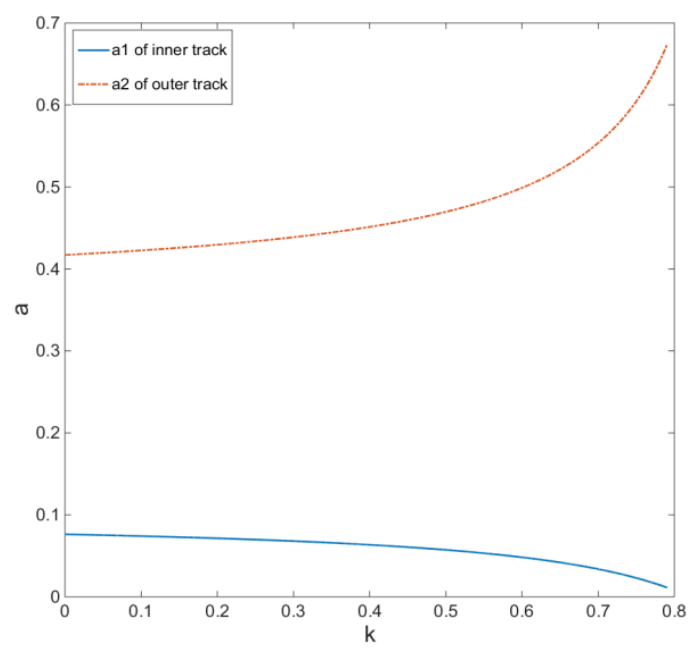

Fig.4 Relationship between $k$ and $a_{1}, a_{2}$

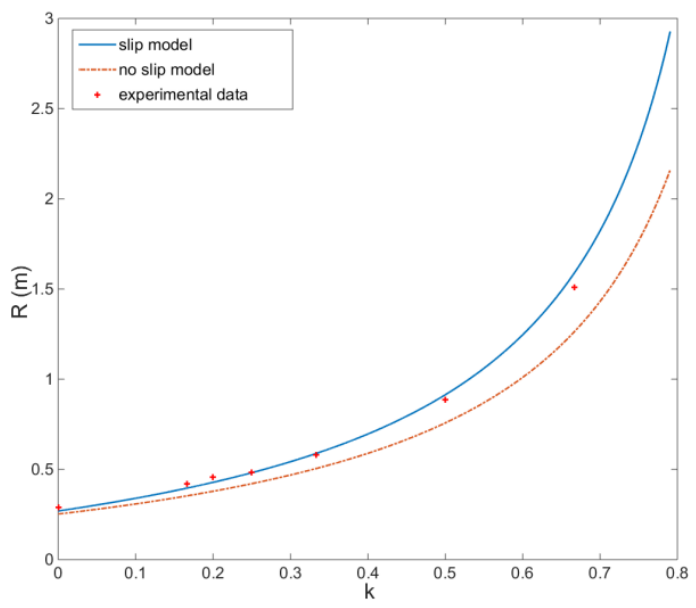

Fig.6 Models of relationship between $k$ and $R$

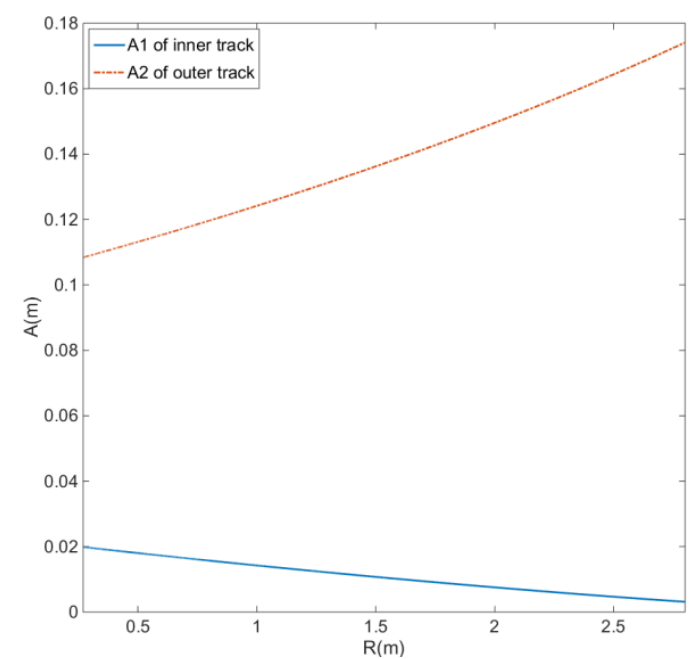

Fig.5 Relationship between $R$ and $A_{1}, A_{2}$

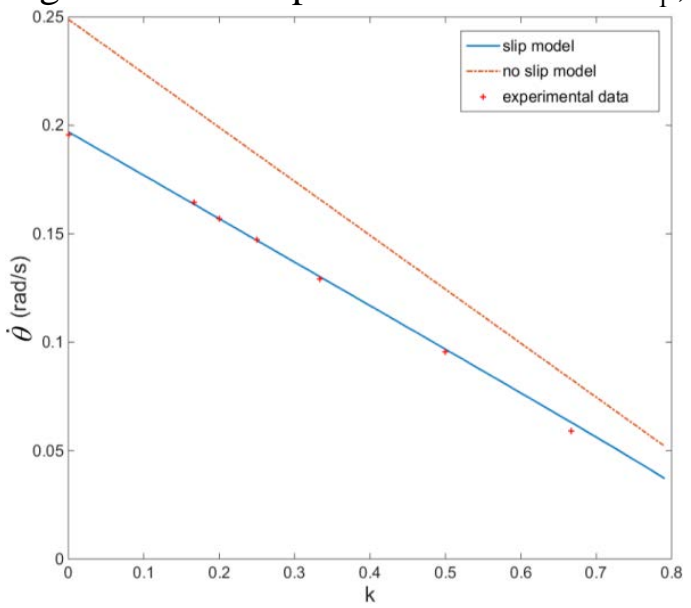

Fig.7 Models of relationship between $k$ and $\dot{\theta}$

\subsection{Experimental Verification.}

To verify the precision of the model, the experiment of TMR steady turning is necessary. In the experiment, the parameters of TMR are in Table 1 . Take $\omega=1.7453 \mathrm{rad} / \mathrm{s}$, then measure the turning radius $R_{e x}$ and the average time of steady turning steering cycle $T$, when control the speed ratio $k$ is varying. The experimental turning angular velocity $\dot{\theta}_{e x}$ can be calculated by formula $\dot{\theta}_{e x}=2 \pi / T$. The comparison of numerical analysis results of slip model and no slip model with experimental data are shown in Table 2 , Fig.6 and Fig.7 .

From Fig.5 and Fig.6, we can find that:

(1) Slip model is more in line with the experimental data compare to no slip model;

(2) At the same $k$, no slip model has smaller turning radius and bigger turning angular velocity comparing to slip model and the experimental data, this is due to the model do not consider the tracks' slipping. The tracks' slipping makes the effective rotating linear velocity of tracks lower, so slip model is of better accuracy. 
Table 2 Comparison of Numerical Analysis Results with Experimental Data

\begin{tabular}{ccccccc}
\hline$k$ & $R_{e x}(m)$ & $R(m)$ & $R_{t}(m)$ & $\dot{\theta}_{e x}(\mathrm{rad} / \mathrm{s})$ & $\dot{\theta}(\mathrm{rad} / \mathrm{s})$ & $\dot{\theta}_{t}(\mathrm{rad} / \mathrm{s})$ \\
\hline 0 & 0.290 & 0.270 & 0.253 & 0.1956 & 0.1970 & 0.2488 \\
0.167 & 0.421 & 0.396 & 0.354 & 0.1643 & 0.1636 & 0.2074 \\
0.200 & 0.458 & 0.428 & 0.378 & 0.1569 & 0.1570 & 0.1991 \\
0.250 & 0.482 & 0.482 & 0.421 & 0.1474 & 0.1469 & 0.1866 \\
0.333 & 0.582 & 0.588 & 0.505 & 0.1291 & 0.1303 & 0.1659 \\
0.500 & 0.885 & 0.914 & 0.758 & 0.0956 & 0.0968 & 0.1244 \\
0.667 & 1.510 & 1.588 & 1.263 & 0.0590 & 0.0631 & 0.0829 \\
\hline
\end{tabular}

From Table 2, we can calculate that the average relative error rate of slip model is $4.14 \%$ for turning radius and $1.49 \%$ for turning angular velocity, while the quantities of no slip model are $14.71 \%$ and $29.45 \%$.

\section{Conclusion}

This paper aims to establish a turning model of TMR under steady state conditions, which considers the tracks' slipping, and is more suitable for TMRs' motion control. Kinematics and dynamics of TMR were analyzed based on the follow-up coordinate system and the ground coordinate system, and the slip model is established based on these analyses. By the comparison of numerical analysis results and experimental data, conclusions can be summarized as follow:

(1) Slip model is more in line with the experimental data compare to no slip model;

(2) At the same $k$, no slip model has smaller turning radius and bigger turning angular velocity comparing to slip model and the experimental data.

(3) The average relative error rate of slip model is $4.14 \%$ for $R$ and $1.49 \%$ for $\dot{\theta}$, while the quantities of no slip model are $14.71 \%$ and $29.45 \%$.

\section{References}

[1]. Moosavian S A A, Kalantari A. Experimental slip estimation for exact kinematics modeling and control of a Tracked Mobile Robot[C]// IEEE/RSJ International Conference on Intelligent Robots and Systems. 2008:95-100.

[2]. Zhou B, Han J, Dai X. Backstepping Based Global Exponential Stabilization of a Tracked Mobile Robot with Slipping Perturbation[J]. Journal of Bionic Engineering, 2011, 8(1):69-76.

[3]. Gao J, Zhang S L. Path tracking control of Micro-Tracked mobile robot[J]. Applied Mechanics \& Materials, 2014, 644:265-271.

[4]. Kabanov Aleksey, Kramar Vadym. Modeling and Control of Tracked Mobile Robot[C]//50 ${ }^{\text {th }}$ ANNIVERSARY TECHNICAL UNIVERSITY OF VARNA. 2012:84-86.

[5]. Xuan V H, Ha C, Choi H S. Path Tracking Control of a Mobile Robot by Using Dual Estimation Algorithm[J]. Advances in Mechanical Engineering, 2015, 5(5):367127-367127.

[6]. Junwei Cheng, Lianhua Gao, Hongyan Wang. Analysis of Tracked Vehicles Steering Based on Conditions of Tracks’ Slip[J]. Journal of Mechanical Engineering, 2006(S1):192-195.

[7]. Wong J Y, Chiang C F. A general theory for skid steering of tracked vehicles on firm ground[J]. Proceedings of the Institution of Mechanical Engineers Part D Journal of Automobile Engineering, 2001, 215(3):343-35. 